\title{
Risk Factors for Obesity and High Blood Pressure in Chinese American Children: Maternal Acculturation and Children's Food Choices
}

\author{
Jyu-Lin Chen · Sandra Weiss · Melvin B. Heyman • \\ Robert Lustig
}

Published online: 16 September 2009

(c) The Author(s) 2009. This article is published with open access at Springerlink.com

\begin{abstract}
The objective of this study is to explore risk factors associated with overweight and high blood pressure in Chinese American children. Students and their parents were recruited from Chinese language schools in the San Francisco Bay Area. Data were collected on 67 children and their mothers, and included children's weight, height, waist and hip circumferences, blood pressure, level of physical activity, dietary intake, usual food choice, knowledge about nutrition and physical activity, and selfefficacy regarding diet and physical activity. Mothers completed questionnaires on demographic data and acculturation. About $46 \%$ of children had a body mass index exceeding the 85 th percentile. Lower level of maternal acculturation is a risk factor for overweight and higher waist to hip ratio. Children's unhealthy food choices were predictive of high body mass index and high systolic blood pressure, whereas older age and less physical activity in children were predictors of high diastolic blood pressure. Developing culturally sensitive and developmentally appropriate interventions to reduce overweight and high
\end{abstract}

\section{J.-L. Chen ( $\square)$}

Department of Family Health Care Nursing, School of Nursing, University of California, San Francisco, 2 Koret Way, Box 0606, San Francisco, CA 94143, USA

e-mail: jyu-lin.chen@nursing.ucsf.edu

\section{S. Weiss}

Department of Community Health Systems, School of Nursing, University of California, San Francisco, 2 Koret Way, Box 0606,

San Francisco, CA 94143, USA

M. B. Heyman · R. Lustig

Department of Pediatrics, School of Medicine,

University of California, San Francisco,

2 Koret Way, Box 0606, San Francisco, CA 94143, USA blood pressure is critical to reduce health disparities among minority children.

Keywords Obesity - Blood pressure .

Chinese-American - Children - Maternal acculturation .

Children's food choice

\section{Background}

Chinese Americans are the largest and fastest growing Asian subgroup in the United States, increasing by $48 \%$ between 1990 and 2000 [1]. Chinese Americans, particularly children, are experiencing an unprecedented increase in obesity and type 2 diabetes mellitus. Recent data indicate that $31 \%$ of Chinese Americans 6-11 years old are overweight, with body mass indices (BMIs) exceeding the 85th percentile [2]. At the same BMI, Chinese Americans are at higher risk of developing cardiovascular disease and type 2 diabetes mellitus than non-Hispanic whites, possibly because of genetic differences in body composition and metabolic responses [3, 4]. Excess weight in children is associated with immediate and long-term medical and psychosocial complications, including high blood pressure, cardiovascular diseases, sleep disorders, type 2 diabetes mellitus, low self-esteem, and social withdrawal [5-9]. Increased risk for obesity and hypertension can result in premature mortality. The adverse effects of these diseases in childhood persist over time and progress to adult premature cardiovascular disease $[5,10]$. Therefore, it is imperative to identify factors associated with increased risk for obesity and hypertension in childhood, especially in Chinese Americans.

Overweight and obesity in children have been identified as a serious health problem. Several factors, including 
children's levels of physical activity, dietary intake, knowledge about adequate nutrition and physical activity, and self-efficacy on obtaining adequate dietary intake and activity level, and maternal acculturation level, are related to overweight in children [11-14]. The relationship between these factors and children's weight status are inconsistent. For example, Agras and Mascola [11] indicate that food intake and sweet beverage consumption are important risk factors for childhood obesity whereas Chen and Kennedy [12] found not association between food intake and risk for childhood obesity. In addition, few studies have explored how these factors contribute to obesity in Chinese American children.

High blood pressure is also associated with the risk for atherosclerosis and premature cardiovascular disease in both children and adults [15, 16]. Elevated blood pressure in childhood is a predictor of high blood pressure in adulthood [17]. Several factors are thought to be related to increased blood pressure in children, including high BMI, lower level of physical activity, high sedentary activity time, and unhealthy dietary intake [18-20]. Lazarou and associates [21] found that higher BMI and frequent eating while watching television are positively related to increased systolic and overall blood pressure in 10- to 13-year-old children. In adolescents, high BMI and more sedentary activity are positively correlated with increased blood pressure [22]. In obese children, increased television viewing time is associated with hypertension [22]. Chinese Americans are at higher risk than non-Hispanic whites for development of cardiovascular disease and atherosclerosis, but little is known about risk factors associated with increased blood pressure in children.

Given the increasing population of Chinese Americans in the United States and the significant impact of overweight and cardiovascular diseases on the health of Chinese American children, a better knowledge of what constitutes or contributes to overweight and high blood pressure can help researchers, health care providers, and the community develop culturally appropriate, gendersensitive, and developmentally based interventions to improve the physical and psychosocial well-being of such children and their families. We sought to explore risk factors associated with overweight and high blood pressure in Chinese American children.

\section{Methods}

\section{Participants}

This protocol was approved by the University of California, San Francisco Committee on Human Research. A randomized experimental design was used to examine the effect of a child-centered and family-focused behavioral intervention in improving health behavior, psychosocial function, and body composition of Chinese American children, 8-10 years old of both sexes. Participants were recruited from Chinese language schools in the San Francisco Bay area. The research assistants described the study to potential students and gave them a letter of introduction and a research consent form to take home to their parents. Parents who were interested in the study signed and returned the consent form, providing their names and contact information to the research team. For the purpose of the paper, we analyzed only baseline data.

\section{Data Collection}

After informed consent had been obtained from parents and verbal assent from children, baseline data were collected. The children and parents were then randomly assigned to the intervention group or to the waiting list control group by means of a computer-generated random number assignment. The intervention consisted of a 1-h smallgroup session (group of 4-6 children) each week for 8 weeks for children and two 2-h small-group sessions for parents. Baseline data were collected 1 week after they were assigned to the study group. Follow up data were collected at 3 and 6 months after the intervention. Children in the waiting list control group received the same intervention after they completed all the follow-up data collection.

The procedures for this study entailed administering questionnaires and taking several physiological measurements. The children and their primary caregiver (all mothers) completed all questionnaires at home and returned them in sealed envelopes within 2 weeks of recruitment into the study. Questionnaires for mothers have been translated into Chinese and demonstrated adequate validity and reliability [23, 24]. Children completed questionnaires in English.

\section{Parental Measures}

\section{Family Information}

This 12-item parent questionnaire includes parent(s)' and children's ages, parents' weight and height, parents' occupation(s), family income, and parents' levels of education. The questionnaire was written at a third-grade reading level and took $\sim 5 \mathrm{~min}$ to complete.

\section{Suinn-Lew Asian Self-Identity Acculturation Scale}

The Suinn-Lew Asian Self-Identity Acculturation Scale (SL-ASIA) was used to examine levels of acculturation 
[23, 24]. The SL-ASIA scale is a 21-item multiple-choice questionnaire covering topics such as language (4 items), identity (4 items), friendships (4 items), behaviors (5 items), general and geographic background (3 items), and attitudes ( 1 item). Scores can range from a low of 1.00, indicative of low acculturation or strong Asian identity, to a high of 5.00, indicative of high acculturation or strong Western identity. The scale also permits classification as "bicultural," indicating that a person has adopted some Asian values, beliefs, and attitudes along with some Western values, beliefs, and attitudes. Validity and a moderate to good reliability $(0.79-0.91)$ also have been reported for Chinese Americans [23, 24].

\section{Children's Measures}

\section{Anthropometric Measures}

After parental consent and the child's assent had been obtained, the research assistants measured the child's body weight and height; the children wore lightweight clothes and no shoes. The Seca 214 Road Rod (Vogel \& Halke GmbH \& Co, Hamburg, Germany) portable stadiometer, which has an excellent graduation of $1 / 8$ inch $(0.1 \mathrm{~cm})$, was used to measure height. Children were instructed to have their head positioned in the Frankfort horizontal plane and to inhale; stretch height was then measured. Body weight was measured by using the 840 Bella Digital Scale (Vogel \& Halke $\mathrm{GmbH} \& \mathrm{Co}$ ), which has a graduation of $0.2 \mathrm{lb}(100 \mathrm{~g})$. Scales were calibrated on the basis of the instructions provided by the companies before each subject was measured. BMI was calculated by dividing body mass in kilograms by height in meters squared. BMI sensitivity ranged from 29 to $88 \%$, specificity ranged from 94 to $100 \%$, predictive value ranged from 90 to $100 \%$, and efficiency ranged from 85 to $100 \%$ in children [25].

In this study, body weight and height were measured 3 times, and the mean values of the three measurements were used to determine BMI. BMIs lower than the 5th percentile were defined as underweight, BMIs between the 6 and 84th percentile were defined as normal weight, and BMIs above the 85 th percentile were defined as overweight, based on the growth chart developed by Centers for Disease Control and Prevention (CDC) [6].

\section{Waist to Hip Ratio}

The waist to hip ratio was derived from waist and hip circumferences. Waist circumference was measured midway between the lowest rib and the superior border of the iliac crest. Hip circumference was measured at the maximal protrusion of the buttocks. The circumferences are given as the mean of the two measurements to the nearest $0.1 \mathrm{~cm}$.

\section{Blood Pressure}

Systolic blood pressure (SBP) and diastolic blood pressure (DBP) were measured by using a mercury sphygmomanometer (Baumanometer; W. A. Baum Co, Copiague, New York) to the nearest $2 \mathrm{~mm} \mathrm{Hg}$, twice on the right arm, with the child seated after $10 \mathrm{~min}$ rest.

\section{Three-Day Food Diary}

This quantitative self-report food diary was used to estimate dietary intake of children. Children received a 3-day food diary containing an instruction sheet, a sample completed day's food-record sheet and 8 blank white dietary record forms. Each form included spaces for the child's name, day of the week, date of recording, and blank lines on which to record food and drink grouped into the following categories: breakfast, snack, lunch, snack, dinner, and snack. Children were asked to record all foods and beverages consumed for 3 days ( 2 weekdays and 1 weekend day). They also recorded serving sizes. The food and beverages were recorded in either Chinese or English. The diets were analyzed for their energy intake, percentage of fat intake, number of fruit and vegetable intake using Nutritionist Pro. Nutritionist Pro has over 20,000 foods and ingredients including brand-name foods, fast foods, and ethnic foods. The nutrient composition of ethnic foods not found in the Nutritionist Pro was obtained from one of the four food composition tables developed for Asian foods (Handbook No. 8. United States Department of Agriculture; Journal of Food composition and Analysis, Special issue: Chinese food composition tables, 1990; Nutrient composition of Malaysia Foods, 1988; Guo-ming yingyang cho dao so chih, National nutrition guidelines, Republic of China, 1991). When the nutrient values for a prepared dish are not available, they were estimated from recipes obtained from recently published recipe books.

This measure has been used in a pediatric population and has adequate reliability. Kappa coefficients and percentage of agreement for interobserver reliability ranged from 0.43 to 0.91 [26]. The diary takes $\sim 10 \mathrm{~min}$ to complete. Parents were asked to complete the diary together with their children to increase the accuracy of dietary report.

\section{Usual Food Choices}

This 14-item survey was part of the Health Behavior Questionnaire developed for the Child and Adolescent Trial for Cardiovascular Health study [27]. This survey asked about usual food choices (behavior) in a forced-choice format that focuses on low-fat and low-sodium foods. It measured usual food selections and what types of food a 
child eats most of the time. Children were given a choice between 2 foods and asked which one they eat more often. Sample questions are "Which foods do you eat most of the time: hot dog or chicken? Frozen yogurt or ice cream?" A higher score indicated more healthy food choices. Validity was obtained by including expert review and focus group pilot testing. The Cronbachalpha coefficient for internal consistency in the original study was 0.76 [27].

\section{Caltrac Personal Activity Computer}

The Caltrac Personal Activity Computer (CPA) has been widely used to assess physical activity among children and adults [28]. It has replaced the pedometer in physical activity research because of its relatively low cost (\$70) and ease of use. The CPA is designed to be placed at the hip and to measure vertical acceleration. Readings from the device have been used to predict oxygen consumption and net caloric expenditure, based on gender, age, height, and weight, during exercise. The CPA indicates a moderate to high validity, ranging from 0.35 to 0.97 , with heart rate and observation methods [28]. A high reliability of the CPA, ranging from 0.87 to 0.98 , was also reported in children [28]. In our study, children placed the CPA at the hip for five consecutive days including one weekend and removed during showers and sleep. Average count was used for analysis.

\section{Dietary Behavior Self-Efficacy}

This 15-item self-report questionnaire measures children's self-confidence in their ability to choose foods low in fat and sugar [27]. The questionnaire contains 15 -item stems beginning with "How sure are you...?" Items are scored on a Likert scale, with options of "not sure," "a little sure," or "very sure." Higher scores indicate higher self-efficacy. The internal consistency ranged from 0.82 to 0.87 in third and fifth graders [27]. It takes about 5 or $10 \mathrm{~min}$ for children to complete the questionnaire.

\section{Physical Activity Self-Efficacy}

This subscale of the Health Behavior Questionnaire is used to measure children's self-confidence in their ability to participate in various age-appropriate physical activities [27]. The subscale includes 5 items in which children are asked if they are "not sure," "a little sure," or "very sure" that they can do such things as "keep up a steady pace without stopping for 15-20 min." Higher scores indicate higher self-efficacy. Internal consistency ranged from 0.67 to 0.69 in third and fifth graders [27]. It takes about 2-5 min for children to complete the questionnaire.

\section{Dietary Knowledge}

This 14-item survey also was part of the Health Behavior Questionnaire developed for the Child and Adolescent Trial for Cardiovascular Health [27]. It measures children's knowledge about healthy food choices. Children were asked to identify which food was "better for your health." Samples of two choices included "whole wheat or white bread" and "frozen corn or canned corn." Content validity was examined by including expert review and focus group pilot testing from the Child and Adolescent Trial for Cardiovascular Health. This survey had a reported internal consistency with a Cronbach alpha ranging from 0.76 to 0.78 [27]. A higher score indicates more accurate dietary knowledge.

\section{Physical Activity Knowledge}

This 5-item questionnaire was developed by the researcher to assess children's knowledge regarding physical activity. Items were adapted from recommendations from the US Department of Agriculture [29] and the American Heart Association [30] regarding dietary guidelines, MyPyramid, and children's health. Sample questions included the following: How much aerobic activity is required for a healthy heart? How many $h$ a day should a child watch television or play video games? The reliability coefficient for internal consistency with sample of children in this study was 0.65 . Children received 1 point for every question they answered correctly. A higher score indicates more accurate knowledge about physical activity needs. The total score was used for analysis.

\section{Data Analysis}

Appropriate descriptive statistics, means, and standard deviations for the quantitative variables, as well as frequencies and percentages for categorical variables, were calculated for all of the study variables. To examine gender difference, chi-square tests were performed for categorical data, and $t$ tests were used for continuous data. Multiple linear regressions were performed on children's BMI, waist to hip ratio, diastolic blood pressure, and SBP. Factors entered into models included children's age, gender, parental acculturation level, physical activity, food choice, self-efficacy related to physical activity, self-efficacy related to dietary behavior, average calorie intake, percentage of fat intake, percentage of sugar intake, and number of fruits and vegetables intake. All statistical analyses were performed with SPSS 15.0 for Windows. Data are expressed as mean $\pm \mathrm{SD}$. Level of significance was set at $P<0.05$. 


\section{Results}

\section{Demographic Characteristics}

Sixty-seven 8- to 10-year-old Chinese American children and their families participated in the study. Approximately $57 \%$ of the children were boys $(n=38)$. Thirty-five children $(52 \%)$ were randomized to the intervention group. The mean age of the children was $8.9 \pm 0.9$ years, of the mothers was $41.4 \pm 4.4$ years, and of the fathers was $44.3 \pm 5.3$ years. Most of the mothers were married $(n=55,82 \%)$. The mean years of education was 14 (SD, 4.5) for mothers and 15.6 (SD, 3.7) for fathers. Approximately $70 \%$ of the families had an annual household

Table 1 Selected personal attributes, dietary and physical activity characteristics among study participants

\begin{tabular}{lcc}
\hline Characteristic & Mean & SD \\
\hline Maternal acculturation mean score & 2.38 & 0.69 \\
Child's age (years) & 8.97 & 0.89 \\
Body mass index $^{\mathrm{a}}$ & 19.03 & 3.22 \\
Waist to hip ratio & 0.89 & 0.05 \\
Systolic blood pressure (mm Hg) & 104.5 & 11.2 \\
Diastolic blood pressure (mm Hg) & 61.0 & 12.5 \\
Physical activity (count) & $3,782.3$ & $1,434.4$ \\
Food choice score & 9.0 & 2.16 \\
Percentage of fat consumption & 31.6 & 12.4 \\
Sugar (g) & 23.4 & 9.0 \\
Servings of vegetables and fruit & 3.9 & 3.1 \\
Nutrition self-efficacy & 2.43 & 0.41 \\
Physical activity self-efficacy & 2.32 & 0.47 \\
Nutrition knowledge & 9.58 & 2.72 \\
Physical activity knowledge & 3.63 & 1.07 \\
\hline a Calculated as the weight in kilograms & divided by the height in \\
meters squared & &
\end{tabular}

income greater than $\$ 40,000$. Demographic characteristics did not differ between the intervention and control groups.

The mean BMI was $19.0 \pm 3.2$ and $\sim 46 \%$ of children had a BMI exceeding the 85 th percentile $(n=31)$. Of the overweight children, $58.1 \%$ were boys. Our data suggest children in our study have a higher rate of obesity than the average. No difference was found between overweight status between boys and girls. The mean waist to hip ratio was $0.89 \pm 0.05$, mean $\mathrm{SBP}$ was $104.5 \pm 11.2 \mathrm{~mm} \mathrm{Hg}$, and mean DBP was $61.0 \pm 12.5 \mathrm{~mm} \mathrm{Hg}$. No difference was found between overweight and normal-weight children (Table 1).

Factors Associated with BMI, Waist to Hip Ratio, and Blood Pressure

Pearson correlation coefficient was used to examine the correlation between independent variables (age, gender, maternal acculturation, children's physical activity, dietary intake, knowledge about adequate nutrition and physical activity, and self-efficacy on diet and physical activity) and dependent variables (BMI, waist to hip ratio, SBP, and DBP). Intake of fruits and vegetables was negatively associated with waist to hip ratio $(r=-0.43, P=0.04)$. In addition, higher SBP and DBP were also associated with higher waist to hip ratio $(r=0.59, P=0.005)$. Lower lever of physical activity was associated with high DBP ( $r=-0.34, P=0.01)$. No factor was associated with SBP and BMI.

Multiple linear regressions were performed on children's BMI, waist to hip ratio, SBP, and DBP. To ensure that the model met the assumptions of linearity, normality, and constant variance, we performed visual scatter plots, standardized residuals, studentized residuals, and Cook distance tests. The data met the model's assumptions. The results suggest that lower level of maternal acculturation $\left(\mathrm{sr}^{2}=0.35, P=0.001\right)$ and unhealthy food choice in

Table 2 Risk factors for increased BMI, waist to hip ratio, SBP, and DBP

\begin{tabular}{|c|c|c|c|c|c|c|}
\hline Outcome & Predictor & $R^{2}$ & $\beta$ & $95 \% \mathrm{CI}$ & $\mathrm{sr}^{2}$ & $F(P)$ \\
\hline \multirow[t]{3}{*}{ Body mass index $(\mathrm{BMI})^{\mathrm{a}}$} & Overall & 0.47 & & & & $10.75(0.001)$ \\
\hline & Acculturation & & -0.59 & $-4.24,-1.34$ & 0.35 & $15.71(0.001)$ \\
\hline & Food choice & & -0.40 & $0.15,1.10$ & 0.16 & $7.24(0.01)$ \\
\hline \multirow[t]{2}{*}{ Waist to hip ratio } & Overall & 0.35 & & & & $7.90(0.01)$ \\
\hline & Acculturation & & -0.59 & $0.008,0.060$ & 0.35 & $7.90(0.01)$ \\
\hline \multirow[t]{2}{*}{ Systolic blood pressure (SBP) } & Overall & 0.16 & & & & $4.59(0.04)$ \\
\hline & Food choice & & -0.40 & $0.07,3.79$ & 0.16 & $4.59(0.04)$ \\
\hline \multirow[t]{3}{*}{ Diastolic blood pressure (DBP) } & Overall & 0.45 & & & & $9.36(0.001)$ \\
\hline & Age & & 0.68 & $3.95,12.17$ & 0.40 & $16.48(0.001)$ \\
\hline & Physical activity & & -0.49 & $-0.006,-0.001$ & 0.20 & $8.43(0.008)$ \\
\hline
\end{tabular}

${ }^{a}$ Calculated as the weight in kilograms divided by the height in meters squared 
children $\left(\mathrm{sr}^{2}=0.16, P=0.01\right)$ were predictors of a high BMI in Chinese American children $\left(R^{2}=0.47\right.$, $F=10.75, P=0.001$ ). A lower level of maternal acculturation was also a predictor of higher waist to hip ratio $\left(R^{2}=0.35, F=7.90, P=0.01\right)$. Unhealthy food choice was a predictor of high $\operatorname{SBP}\left(R^{2}=0.16, \quad F=4.59\right.$, $P=0.04)$, whereas older age and a lower level of physical activity were predictors of high DBP $(R=0.45, F=9.36$, $P=0.001$; Table 2).

\section{Discussion}

The prevalence of childhood obesity and high blood pressure has increased dramatically in the past two decades, so understanding the factors related to increased risk for obesity and high blood pressure is critical, especially in a fast-growing population of immigrants. Our study results suggest that a lower maternal acculturation level and unhealthy food choices are risk factors for increased BMI in Chinese American children. Additionally, making unhealthy food choices is also a risk factor for increased SBP, whereas a low level of physical activity and older age are risk factors for increased diastolic blood pressure in Chinese American children.

Studies have suggested that low level of maternal acculturation is associated higher BMI in children [31, 32]. It is possible that mothers with higher levels of acculturation are more sensitive about their own child's weight status because they are more aware of the health issues associated with childhood obesity and therefore are more likely to provide healthy food and encourage active lifestyle [32, 33]. In addition, acculturated mothers may also have better access to healthier food than those who are less acculturated as higher acculturation is associated with higher level of household income [34]. Future study need to examine the mechanisms by which mothers' acculturation may affect proximal determinants of energy balance and overweight risk in children, including dietary intake, and physical activity.

Another important factor associated with obesity and high blood pressure is children's food choices. Our study indicates that choosing unhealthy foods is a risk factor for increased BMI and high SBP. This is consistent with other study that suggests unhealthy dietary intake, especially sugar-sweetened beverage, is associated with increased blood pressure in children [35]. In addition, increased fruit and vegetable intake is related to lower waist to hip ratio in Chinese Americans. An important aspect of children's health behaviors is dietary behavior [36, 37]. Overweight children and children at risk for becoming overweight report a higher preference for high-fat foods and more patterns of overeating than do normal-weight children [35,
38]. Several studies that used dietary interventions have demonstrated successful weight reduction in overweight and obese children [39, 40]. Additionally, a meta-analysis of eight randomized controlled trials in a recent systematic review of the effectiveness of dietary interventions in overweight children indicated that interventions that contain a dietary component are effective in weight management in overweight children and adolescents (standardized mean difference -1.82 with $95 \%$ confidence interval $[\mathrm{CI}]=-2.4$ to -1.23 ) [41]. Therefore, a critical element of a program for preventing overweight and cardiovascular disease in Chinese American children should incorporate healthy eating.

Our results indicate that decreased physical activity and older age are risk factors for increased diastolic blood pressure. Regular physical activity may decrease the prevalence of overweight, death from cardiovascular diseases, diabetes, and high cholesterol [42-44]. A lower level of physical activity is related to increase the risk for a higher level BP in children [45, 46]. A low physical activity level and high BMI were independently associated with a high BP and risk of having hypertension in both genders [45]. Our results are consistent with the above mentioned studies and suggest that low lever of physical activity put children at higher risk for high blood pressure and CVD risks. Thus, increased physical activity is critical in CVD risk reduction in children.

However, Asian children engage in lower levels of physical activity than do white and African American children [47]. The lower level of physical activity and high inactive level may reflect a cultural difference. The low levels of activity may be related to the emphasis placed on academic achievement of children in Asian cultures; as a result, these children spend more time studying or reading than being physically active [48]. Moreover, new immigrant families may also be unaware of the health benefits of physical activity and thus may not promote physical activity.

A study indicated that children's levels of physical activity decline with age (at about age 13), that girls' levels of physical activity decline with age more rapidly than do boys' levels of physical activity, and that boys spend more time engaging in physical activity than do girls [49]. Healthy People 2010 emphasizes the importance of regular participation in moderate physical activity as an essential component of a lifestyle that promotes health and has identified physical activity as a leading health indicator [50]. Therefore, physical activity could be an integral component of children's health behaviors and of the prevention of overweight and cardiovascular disease in children, especially in Chinese Americans.

The results of this study of factors contributing to obesity and high blood pressure in Chinese American 
children should be interpreted carefully. Because of the cross-sectional design of this analysis, no causal relationship can be established. A longitudinal study is needed to examine the change in children's body composition and blood pressure and the factors contributing to these changes. Moreover, because the study involved many selfreported measures, errors in measurement such as under or estimate food consumption may have occurred. Because the children and families who participated in this study are well educated, results can be generalized only to a similar population. Future studies should include children and families who are from different locations and educational backgrounds.

Although results indicated that a small to moderate percentage of variance was explained by the factors we examined in this study, a larger amount of variance was still unexplained. Future studies should incorporate other important factors, such as parental BMI, health perception regarding obesity and cardiovascular disease, and barriers to a healthy lifestyle.

\section{Conclusions}

In this study, we found that lower maternal acculturation level and unhealthy food choices are risk factors for obesity and high blood pressure. As excess weight in children is associated with immediate and long-term medical and psychosocial complications, and the social and economic costs of overweight and of attempts to prevent or to treat overweight are high, interventions targeted to reduce overweight and high blood pressure in children are critical. Promoting healthy weight and preventing overweight in Chinese American children are crucial first steps to reducing health disparities among minority and underserved children.

Acknowledgments This publication was made possible by grant number KL2 RR024130 to J.L.C from the National Center for Research Resources, a component of the National Institutes of Health (NIH) and NIH Roadmap for Medical Research, and in part by NIH grant DK060617 to M.B.H.

Open Access This article is distributed under the terms of the Creative Commons Attribution Noncommercial License which permits any noncommercial use, distribution, and reproduction in any medium, provided the original author(s) and source are credited.

\section{References}

1. Census R. Census 2000 releases national statistics on foreignlanguages spoken in US households: Chinese now ranked \#2 in the nation after Spanish, up from \#4 in 1990. Four of top-10 languages now spoken in US are Asian. 2000.
2. Tarantino R. Addressing childhood and adolescent overweight. Paper presented at the NICOS meeting of the Chinese Health Coalition. San Francisco. 2002.

3. Stevens J. Ethnic-specific revisions of body mass index cutoffs to define overweight and obesity in Asians are not warranted. Int $\mathbf{J}$ Obes Relat Metab Disord. 2003;27(11):1297-9.

4. Tan CE, et al. Can we apply the national cholesterol education program adult treatment panel definition of the metabolic syndrome to Asians? Diabetes Care. 2004;27(5):1182-6.

5. Buiten C, Metzger B. Childhood obesity and risk of cardiovascular disease: a review of the science. Pediatr Nurs. 2000;26(1):13-8.

6. Centers for Disease Control and Prevention. Childhood obesity. 2007.

7. Datar A, Sturm R, Magnabosco JL. Childhood overweight and academic performance: national study of kindergartners and firstgraders. Obes Res. 2004;12(1):58-68.

8. Friedland $\mathrm{O}$, et al. Obesity and lipid profiles in children and adolescents. J Pediatr Endocrinol Metab. 2002;15(7):1011-6.

9. Swallen KC, et al. Overweight, obesity, and health-related quality of life among adolescents: the national longitudinal study of adolescent health. Pediatrics. 2005;115(2):340-7.

10. Goran MI, Ball GD, Cruz ML. Obesity and risk of type 2 diabetes and cardiovascular disease in children and adolescents. J Clin Endocrinol Metab. 2003;88(4):1417-27.

11. Agras WS, Mascola AJ. Risk factors for childhood overweight. Curr Opin Pediatr. 2005;17(5):648-52.

12. Chen JL, Kennedy C. Factors associated with obesity in ChineseAmerican children. Pediatr Nurs. 2005;31(2):110-5.

13. Eisenmann JC. Physical activity and cardiovascular disease risk factors in children and adolescents: an overview. Can J Cardiol. 2004;20(3):295-301.

14. Eisenmann JC, Bartee RT, Wang MQ. Physical activity, TV viewing, and weight in U.S. youth: 1999 youth risk behavior survey. Obes Res. 2002;10(5):379-85.

15. Beilin L, Huang RC. Childhood obesity, hypertension, the metabolic syndrome and adult cardiovascular disease. Clin Exp Pharmacol Physiol. 2008;35(4):409-11.

16. Matsuoka S, Awazu M. Masked hypertension in children and young adults. Pediatr Nephrol. 2004;19(6):651-4.

17. Lauer RM. Childhood predictors for high adult blood pressure. The Muscatine Study. Pediatr Clin North Am. 1993;40(1):23-40.

18. Chiolero A, et al. Has blood pressure increased in children in response to the obesity epidemic? Pediatrics. 2007;119(3):544-53.

19. Couch SC, Daniels SR. Diet and blood pressure in children. Curr Opin Pediatr. 2005;17(5):642-7.

20. Sorof J, Daniels S. Obesity hypertension in children: a problem of epidemic proportions. Hypertension. 2002;40(4):441-7.

21. Lazarou C, Panagiotakos DB, Matalas AL. Lifestyle factors are determinants of children's blood pressure levels: the CYKIDS study. J Hum Hypertens. 2008.

22. Pardee PE, et al. Television viewing and hypertension in obese children. Am J Prev Med. 2007;33(6):439-43.

23. Suinn RM, Khoo G, Ahuna C. The Suinn-Lew Asian self-identity acculturation scale: cross-cultural information. J Multicult Couns Devel. 1995;27:139-48.

24. Suinn RM. Measurement of acculturation of Asian Americans. Asian Am Pac Isl J Health. 1998;6(1):7-12.

25. de Onis M. The use of anthropometry in the prevention of childhood overweight and obesity. Int J Obes Relat Metab Disord. 2004;28(Suppl 3):S81-5.

26. Weber JL, et al. Validity of self-reported dietary intake at school meals by American Indian children: the pathways study. J Am Diet Assoc. 2004;104(5):746-52.

27. Edmundson E, et al. The effects of the child and adolescent trial for cardiovascular health upon psychosocial determinants of diet and physical activity behavior. Prev Med. 1996;25(4):442-54. 
28. Noland M, et al. The measurement of physical activity in young children. Res Q Exerc Sport. 1990;61(2):146-53.

29. Agriculture, U.S.D.o. Steps to a Healthier You. 2006. [cited May 15, 2008]; Available from: http://mypyramid.gov/downloads/ MiniPoster.pdf

30. Association, A.H, editor. Children's health. Dallas: American Heart Association; 2006.

31. Chen JL, Wu Y. Cardiovascular risk factors in Chinese American Children: associations between overweight, acculturation and physical activity. J Pediatr Health Care. 2008;22:103-10.

32. Sussner KM, Lindsay AC, Peterson KE. The influence of maternal acculturation on child body mass index at age 24 months. J Am Diet Assoc. 2009;109(2):218-25.

33. Sussner KM, et al. The influence of immigrant status and acculturation on the development of overweight in Latino families: a qualitative study. J Immigr Minor Health. 2008;10(6): 497-505.

34. Chen JL, Wu Y. Cardiovascular risk factors in Chinese American children: associations between overweight, acculturation, and physical activity. J Pediatr Health Care. 2008;22(2):103-10.

35. Nguyen S, et al. Sugar-sweetened beverages, serum uric acid, and blood pressure in adolescents. J Pediatr. 2009;154(6):807-13.

36. Cottrell L, et al. A kindergarten cardiovascular risk surveillance study: CARDIAC-Kinder. Am J Health Behav. 2005;29(6): 595-606.

37. Gunnarsdottir I, Thorsdottir I. Relationship between growth and feeding in infancy and body mass index at the age of 6 years. Int J Obes Relat Metab Disord. 2003;27(12):1523-7.

38. Raynor HA, et al. A cost-analysis of adopting a healthful diet in a family-based obesity treatment program. J Am Diet Assoc. 2002;102(5):645-56.

39. Caballero B, et al. Pathways: a school-based, randomized controlled trial for the prevention of obesity in American Indian schoolchildren. Am J Clin Nutr. 2003;78(5):1030-8.
40. Ernst ND, Obarzanek E. Child health and nutrition: obesity and high blood cholesterol. Prev Med. 1994;23(4):427-36.

41. Collins CE, et al. Measuring effectiveness of dietetic interventions in child obesity: a systematic review of randomized trials. Arch Pediatr Adolesc Med. 2006;160(9):906-22.

42. Deforche B, et al. Role of physical activity and eating behaviour in weight control after treatment in severely obese children and adolescents. Acta Paediatr. 2005;94(4):464-70.

43. Rooney BL, et al. Growing healthy families: family use of pedometers to increase physical activity and slow the rate of obesity. WMJ. 2005;104(5):54-60.

44. Yin Z, et al. Physical activity buffers the effects of chronic stress on adiposity in youth. Ann Behav Med. 2005;29(1):29-36.

45. Nielsen BM, et al. Prediction of fat-free body mass from bioelectrical impedance among 9- to 11-year-old Swedish children. Diabetes Obes Metab. 2007;9(4):521-39.

46. Schmidt G, Walkuski JJ, Stensel DJ. The Singapore Youth coronary risk and physical activity study. Med Sci Sports Exerc. 1998;30:105-13.

47. O'Loughlin J, Paradis G, Renaud L, Meshefedjian G, GrayDonald K. Prevalence and correlates of overweight among elementary schoolchildren in multiethnic, low income, inner city neighborhoods in Montreal, Canada. Ann Epidemiol. 1998;8: 422-32.

48. Chen JL, Kennedy C. Family functioning, parenting style and Chinese children's weight status. J Fam Nurs. 2004;10:186-95.

49. Sanchez Bayle M. A longitudinal study of physical exercise practice in children. The influence of age, gender and socioeconomic level. The working group on cardiovascular risk factors in childhood and adolescence. An Esp Pediatr. 1998;48(1):25-7.

50. US Department of Health and Human Services. Healthy people 2010, 2nd ed. Understanding and improving health and objectives for improving health, 2 vols. Washington, DC: US Government Printing Office; 2000. 